\title{
Investigating the Apparent Link between Cosmic Ray Muon Flux, Sudden Stratospheric Warming and Dry Season Rainfall over Jamaica
}

\author{
Lawrence Patrick Brown ${ }^{1,2 *}$, John Charlery3, Mitko Voutchkov² \\ ${ }^{1}$ Meteorological Service, Kingston, Jamaica \\ ${ }^{2}$ Physics Department, University of the West Indies, Mona, Jamaica \\ ${ }^{3}$ Department of Computer Science, Mathematics and Physics, University of the West Indies, Cave Hill, Barbados \\ Email: ^larro1924@yahoo.com
}

How to cite this paper: Brown, L.P., Charlery, J. and Voutchkov, M. (2019) Investigating the Apparent Link between Cosmic Ray Muon Flux, Sudden Stratospheric Warming and Dry Season Rainfall over Jamaica. Atmospheric and Climate Sciences, 9, 662-682.

https://doi.org/10.4236/acs.2019.94041

Received: February 20, 2019

Accepted: October 25, 2019

Published: October 28, 2019

Copyright ( $\odot 2019$ by author(s) and Scientific Research Publishing Inc. This work is licensed under the Creative Commons Attribution International License (CC BY 4.0).

http://creativecommons.org/licenses/by/4.0/

\begin{abstract}
The Jamaican primary dry season extends from November-April with the driest period being January-March each year. Examination of the rainfall records over several decades reveal months that experienced a sharp increase in rainfall while sometimes they are drier than normal. During this dry season, some of the weather systems that impact the island significantly are cold fronts, highs pressure systems and troughs that migrate from the mainland USA. Major Sudden Stratospheric Warmings (SSW) occur routinely north of $60^{\circ} \mathrm{N}$ and severely impact weather over the North American continent. The islands of the Greater Antilles also experience inclement weather during this period as weather systems migrate southwards and impact the Caribbean. Severe winter weather due to the increase frequency and intensity of storms related to SSW events over North America is important to the Jamaican economy as tourists escape harsh winters by travelling to the island. Predicting the variability of rainfall during the primary dry season is therefore important as it has significant implications for event planning, tourism and agriculture. Cosmic-ray Muon flux has been found to be positively correlated with the atmospheric effective temperature used to indicate the occurrence of SSW events. Current research investigates the relationship between cosmic-ray Muon flux, SSW events and primary dry season rainfall. Our findings suggest that a significant change in rainfall occur over Jamaica during the primary dry season on average 29 days after the central date or on average 15 days after the SSW events end. Our study also suggests apparent similarities in the behaviour of cosmic-ray Muon flux over Jamaica to those when major SSW
\end{abstract}


occurs at high latitudes. We therefore argue that the Muon detectors at low latitudes have practical use with respect to identifying SSWs and merits further study.

\section{Keywords}

Cosmic-Ray Muon Flux, Sudden Stratospheric Warming, Primary Dry Season Rainfall Jamaica

\section{Introduction}

While astronomical seasons have fixed periods based on the position of the Sun with the boreal winter starting December 21 and ending March 20, the meteorological seasons are based on changes in the climatological temperature and cover a portion of the astronomical season. The meteorological winter for example begins December $1^{\text {st }}$ and ends on the last day in February (note here after 3 letters are used to represent months). According to [1], [2] and [3] the period Nov-Apr can be viewed as the primary dry season (PDS) for most of the Caribbean. Examination of the Jamaican rainfall records reveal that some of the driest months in this primary dry season are Jan, Feb and Mar which overlaps with boreal winter. [3] and other authors suggest a link between the equator ward expansion of the North Atlantic High pressure system, subsidence and divergence in the lower-troposphere as the reason for reduced rainfall during the primary dry season (PDS). This argument is supported by the fact that these meteorological processes are used to decide atmospheric stability, which generally leads to dry conditions. However, PDS records covering several decades have shown months that experienced a sharp increase in rainfall while sometimes they are drier than normal.

The behaviour of stratospheric wind and temperature regimes has also been shown to impact tropospheric Jetstreams and results in equatorward shift in the North Atlantic High (NAH). This has at times been accompanied by anomalous cold conditions over the northern hemisphere, [4], [5] and [6]. Sudden Stratospheric Warming (SSW) is a sharp increase in stratospheric temperature usually observed north of $60^{\circ} \mathrm{N}$. While [7] argued the need for a standard definition, [8] defined SSW as the deviation of the polar temperature at $30 \mathrm{mb}$ from its long term climatological mean value. This phenomenon has been found to impact the winds associated with the polar vortex and weather over North America [9]. A polar vortex is an area of very cold air surrounded by strong winds moving cyclonically around the poles. These winds which generally flow from the west are usually weaker during the summer and stronger during the winter, [10]. SSWs are named based on whether they result in a total displacement of the polar vortex away from the poles or on the splitting of the polar vortex into two separate vortices. There can be minor SSWs where the sudden warming occurs and the winds associated with the polar vortex slows down but does not change direc- 
tion, and there can be major SSWs where a sudden warming occurs which is accompanied by a change in the climatological mean wind. The date on which this change in wind direction occurs is known as the central date. [11] defined the central dates of the SSWs as the date that the daily-mean zonal-mean zonal wind at $10 \mathrm{mb} 60^{\circ} \mathrm{N}$ first change from westerly to easterly between Nov and Mar. [12] in their study of the displacement and splitting of the polar vortices noted that the decay stage of the splitting event had the largest impact on weather. During the decay stage surface temperature patterns are similar but the intensity of the signals is not. During the decay stage warm anomalies are observed over eastern North America and cold anomalies over northern Eurasia. One reason offered for this warming or SSW over the Polar Regions is sinking air which results from the energy associated with Ross by waves moving upwards into the stratosphere.

SSWs have also been found to be strongly connected to many other significant weather and climate phenomena. These include the North Atlantic Oscillation (NAO), Arctic Oscillation (AO) and Quasi Biennial Oscillation (QBO), [6]. Authors such as [13], [14], and [15] have explained Caribbean rainfall variability based on the aforementioned phenomena, including the El Nino and Southern Oscillation (ENSO). In many of these studies however, the linkages between tropospheric phenomena such as sea surface temperature anomalies and Caribbean rainfall are explored mainly regarding the wet season [14], [16]. [3] argued however, that very little is known regarding the drivers of rainfall anomalies for the primary dry season and suggested that this is an area that merits much more research. Current study therefore attempts to address this issue. However, [17] informs of another warming of the stratosphere each year referred to as stratospheric final warming (SFW), and argues that there is a strong correlation between the occurrence of the major SSW and the seasonal timing of Stratospheric Final Warmings (SFWs). SFW is defined as the routine annual change in westerlies to easterlies of the polar jet which results in warming. They also argue that the onset date of SSWs varies between late Nov to mid-Mar while the onset period of SFW is usually between mid-Mar and mid-May (average Apr30). The variability in the timing of routine seasonal stratospheric final warming (SFW) has also been found to impact the rainfall and temperature patterns over various regions. Although SFWs are also of interest to us since it has implications for this study, it was not the focus and should not be mistaken with the episodic sudden warming of the stratosphere which defines SSW.

Cosmic-ray Muons are sub-atomic particles that are initially formed from the interactions of cosmic radiation with particles and molecules within the lower stratosphere. The intensity of cosmic-ray Muons reaching the Earth's surface varies due to both thermodynamic processes at the level Muons are first generated and the turbulent processes caused by sudden changes in density [18]. [19] and [20] have shown there is a direct link between SSW and cosmic-ray Muon flux (CMF) via the atmospheric effective temperature. The effective temperature is the weighted-atmospheric temperature that acts as a proxy for the temperature of the real atmosphere when the various conditions from the point of observa- 
tion to the region of Muon generation are considered, [21] and [22]. [23] strengthens our argument of the link between cosmic-ray Muon flux (CMF)-Sudden Stratospheric Warming (SSW) and the primary dry season (PDS) by pointing to a strong statistical correlation between cosmic-ray flux, large-scale precipitation and precipitation efficiency. An examination of the total rainfall over Jamaica revealed that there is a general decrease in rainfall over the last few decades and that stratospheric winter coincides with the primary dry season (Nov-Apr). A review of the literature surrounding SSWs revealed that they only occur during stratospheric winter (Nov-Apr) and that their highest frequency coincides with the driest period of the Jamaican primary dry season (Jan-Mar), which overlaps the boreal winter.

In this paper, we suggest that SSW events are El Nino-type phenomena that have far-reaching impact on our atmosphere and are likely to extend to low latitudes. Our hypothesis is also supported by [24] who argues that SSW affects the whole atmosphere by affecting its chemistry, temperature, wind fields, electron density and non-ionizing particles. The approach taken in this study was to 1) establish a link between the observed SSW events and the Jamaican PDS rainfall, 2) explore the correlation between PDS rainfall and cosmic-ray Muon flux and 3) identify an empirical link between reported SSW at high latitude and cosmic-ray Muon flux measured at low latitudes. This was done against the background that the study will continue as more CMF data are being gathered. The concept of SSW-PDS-CMF relation is therefore discussed in the following sections with background information drawn from a review of the literature in Section 2 while Section 3 will discuss the data and method. In Section 4, the results will be outlined after which we will conclude in Section 5 .

\section{Background}

According to [24], weather events $10-50 \mathrm{~km}$ above in the stratosphere affect weather at the Earth's surface. [24] also argued that General Circulation Model (GCM) projections based on Madden Julian Oscillation forcing, indicate an increased number of SSW events in the future. [25] went further to state that SSW accompanied by changes in the wind direction and wind speeds linked to the polar vortex, can result in significant changes in weather on the ground over eastern regions of the USA. [25] also argues the need for greater accuracy in predicting SSW and points to several studies which used different methods to predict SSWs with fairly good results in 2 - 15 days. Using the SSW frequency records indicated in [9] and [26], it was clear that the amount of SSWs is approaching one event per year, representing an increase from the 6 per decade previously. [27] suggests a link between SSW and the NAO, indicating a negative $6.5 \mathrm{mb}$ fall in $\mathrm{NAO}$ related pressure and an in significant skill in forecasting $\mathrm{NAO}$ when SSW is not included as a predictor in forecast ensemble. An examination of the SSW-PDS profile developed for this study indicated that while the number of SSWs has increased, there has been significant variability in rainfall particularly during the driest period (Jan-Mar). This period according to [28] 
has the highest SSW frequency with an average frequency date of Jan 25th. One potential consequence of severe SSW related winter storms which affect the eastern regions of the USA is an increase in the number transient weather systems such as troughs, cold fronts and highs pressure systems which eventually impact the Caribbean.

Precipitation is one of the most fundamental climate variables due to its importance in atmospheric dynamics and as a natural resource [23]. Many attempts have been made to predict rainfall for the dry and wet season using correlation with climate phenomena such as the ENSO and the NAO. However, [29] after investigating the cosmic-ray Muon flux link between fronts and troughs, argued that there is a connection. In their study [29] used Geiger Muller detectors to measure Muon counts for different 5-hour periods throughout the day and suggest that the cosmic-ray Muon counts were most times inversely correlated to the altitude of the freezing level $\left(0^{\circ} \mathrm{C}\right.$ level $)$. They also observed that when meteorological fronts and troughs were near the detector, a reversal pattern occurs, that is, the graph would show negative slope at the first increment of a date rather than a positive one. They therefore argued that the Muon decay effect or temperature effect, affects the height of Muon production, the intensity and quantity of Muons at sea level. [30] also argues that both increases in the atmospheric pressure and temperature can lead to an inverse correlation to low energy cosmic-ray Muon flux. They argued that increases in atmospheric pressure causes Muons to lose more energy and decay before reaching the surface while increased average temperature causes the atmosphere to expand, resulting in primary cosmic-rays interacting at a higher altitude. This greater travel path therefore increases the probability of Muons decaying before reaching ground based detectors.

Other suggestions have been made regarding large scale weather systems such as tropical cyclones (TC) and cosmic-ray Muon flux. Although TC activity rarely occur during the PDS consideration is given since the hurricane season ends Nov 30th and therefore overlaps with the stratospheric winter season. Furthermore, with our changing climate, the usual start and end times for this season when TCs are active is likely to change. [31] argues that low atmospheric pressure over the Caribbean is important for hurricane development and have shown a connection between hurricanes and cosmic-ray Muon flux. [31] argues further that when the atmospheric pressure is low, there are less air molecules and so cosmic-ray Muons are able to penetrate deeper through the atmosphere, resulting in a surge of ionization near the Earth's surface. During the Main Injector Neutrino Oscillation Search (MINOS) research done deep underground, [19] showed a positive correlation between SSW and cosmic-ray Muon flux using the effective temperature. [20] using the multipurpose detector at the Amundsen-Scott South Pole station (IceCube) arrived at similar results. The effective temperature is an altitude based weighted average temperature where [22] showed that up to twenty one (21) different pressure levels can be used in its calculation. To find the weighted function needed in the calculation of the ef- 
fective temperature a simulation package, such as Geant 4 , is used to determine the Muon counts in the various atmospheric layers. [32] and [33] also linked cosmic-ray Muons to atmospheric temperature and pressure, stating the dynamic steps used to remove their impact onMuon flux to allow its use in the monitoring of extra-atmospheric phenomena.

[3] using canonical analysis, found that while ENSO influences rainfall over the Caribbean and that there was a transition zone $18^{\circ} \mathrm{N}-20^{\circ} \mathrm{N}$ where values changed from positive to negative rainfall anomalies or vice versa, when ENSO impacts were nonexistent. This zone includes Jamaica, Hispaniola and the Cayman Islands, which is also impacted by weather conditions after SSW and also merits further research. [32] also argues that rainfall during Feb-Apr is influenced by sea surface temperatures over the Tropical North Atlantic. The correlation statistics from their study were referred to as robust and are similar to those in this study. Part of the explanation being pursued for dry season rainfall variability in this study is based on kinematic processes related to weather systems after SSW rather than the dynamic processes which link the Jamaican dry season rainfall to SSW. Although empirical evidence would suggest that western Cuba would suffer much greater impact from transient weather systems after SSW events the mean monthly rainfall over Jamaica was used as a representative value for the northwestern Caribbean. Our aim is therefore supported by [32], who argued that other mechanisms should be investigated to explain the variability of Caribbean rainfall as the mechanisms being used to explain rainfall over one season, does not necessarily explain that of other seasons. The need for further research regarding SSW and its likely impact on the Caribbean is therefore being suggested as one alternate and its link to cosmic-ray Muon flux as an exceptional one to those already explored. In this study we investigated the cosmic-ray Muon flux, Sudden Stratospheric Warming, primary dry season rainfall (CMFSSW-PDS) connection. We therefore suggest that the occurrence of major SSWs which usually results in a change in wind direction of the Polar Vortex from westerly to easterlies during the winter, induces the stratospheric "summer effect during winter" and increases the occurrence of rainfall from troughs and fronts as cold air moves further south over the Caribbean. Section 3 below outlines the method used with a brief background before.

\section{Method}

\subsection{Background to Method}

During current study, several data sources were examined, however, three (3) datasets were employed to determine statistical and empirical relationships, 1) the cosmic-ray Muon flux dataset captured at the Mona Campus of the University of the west Indies (UWI Mona), 2) the SSW duration and central dates sourced mainly from [9] and [26], and 3) the rainfall records from stations over the USA and the Caribbean.

The central dates for SSW episodes were from a combination of profiles with 
most coming from the JRA-55 (Japanese 55-year Reanalysis) dataset which allowed for significant long-term analysis of SSW vs Primary Dry Season rainfall. The records of the Reagan International Airport (RIA) in Washington DC, Sangster International Airport (SIA) and Norman Manley International Airport (NMIA) in Jamaica were used to determine correlations between SSW and cosmic-ray Muon flux. RIA was chosen to represent the central to eastern USA, SIA represented northern parishes over Jamaica and NMIA the southern parishes. A daily area average of rainfall for SIA and NMIA was also generated. Figure 1 below illustrates the Caribbean region with the islands referenced in this study.

The high time-scale cosmic-ray Muon observations recorded at the UWI using the QuarkNet 6000 detector were separated into 5-minute bin width. A Muon observation index was then created from an average of the10-minute period that straddles the main hour (that is every 6 hours). This was done over the period Sep 2011 to Sep 2013 and separated into two periods. The first period (Sep 2011 to Oct 2012) was regarded as the control period where we sought to count all the hits on a single channel (channel 2) of the detector. The second period (Nov 2012 to Sep 2013) was the test period where the detector was calibrated for 2 -fold coincidence using channel 1 and channel 2. During the first period all particles hitting the detector was registered along with the thermal noise effects associated with the photomultiplier tubes (PMT). This was referred to as cosmic-ray Muonflux plus or CMF+. Muons are the most abundant sub-atomic particles at ground level with $50 \%-70 \%$ of the particles registered at the surface being Muons, [34], [35]. Therefore the Muon component would have some sensitivity to the atmospheric processes. Using the coincidence method for the second period (Nov 2012 to Sep 2013), it was accepted with more certainty that real cosmic-ray Muons were being registered on the detector [35]. This data was then correlated with rainfall and other station data (some not shown) from

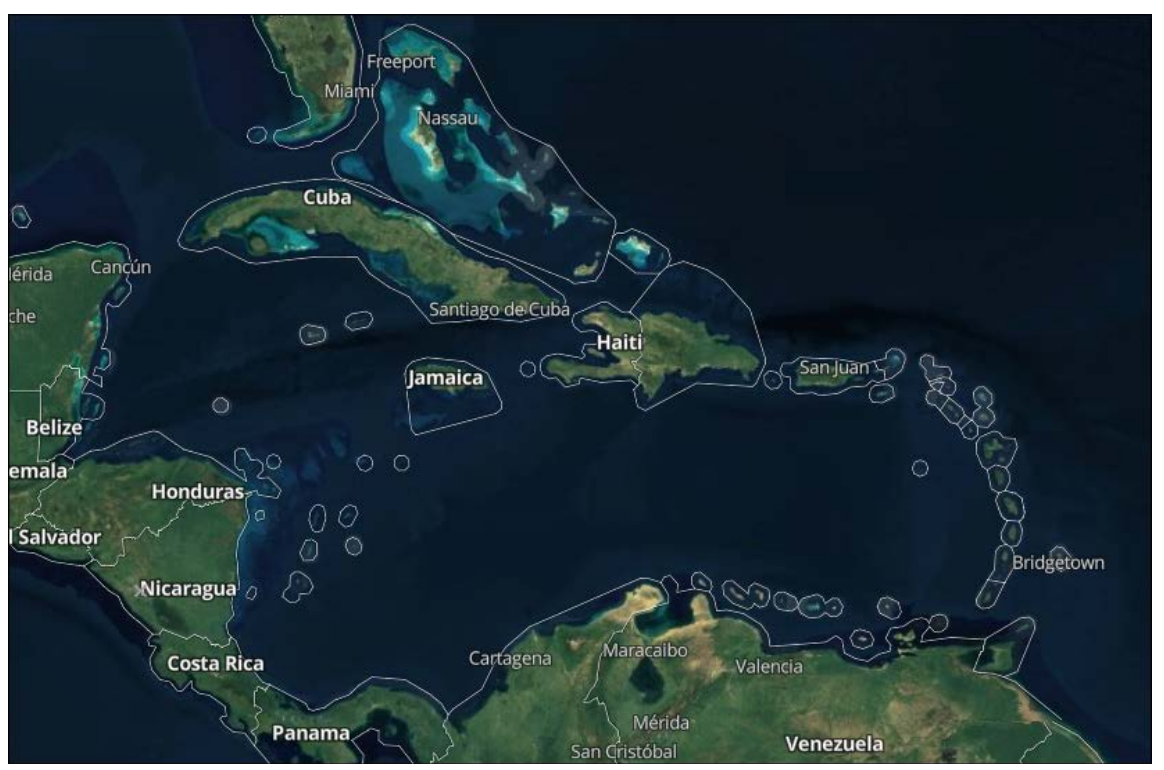

Figure 1. The Caribbean region showing locations where data was gathered. 
Sangster International Airport (SIA) and the Norman Manley International Airport (NMIA). The reports of severe weather over the eastern sections of the USA were drawn from websites hosted mainly by the USA National Weather Service.

\subsection{Method}

The cosmic-ray Muon data captured at the UWI Mona physics department was first quality controlled using both the I2U2.org website and manual examination of the data. Application on the I2U2.orgwebsites was then used to separate data into several bin widths ranging from minutes to hours. After extensive testing to arrive at an appropriate bin width the 5-minute bin width (here after Muon5) was chosen. The Muon5 was then averaged over a 10 minute period with each main hour falling in the middle. The main hours (every 6th hour) were used as an index. These were similar to a synoptic observation times required by the World Meteorological Organization (WMO) and were as follows 0000UTC (Mu00Z), 0600UTC (Mu06Z), 1200UTC (Mu12Z) and 1800 UTC (Mu18Z). An extensive assessment and correlation analysis was then carried out between these daily and monthly muonic indices, rainfall, and SSW events. The daily muonic indices were also matched against the period when SSW events occurred during the primary dry season. The following assumptions were made to guide our method and to arrive at our conclusions

1) Since the stratospheric winter period, boreal winter and Jamaica's primary dry season coincided, there is a high probability that there is a one or phenomenon impacting all. This is especially since the driest months of the primary dry season was Jan-March which are the months when SSWs were most frequent and any guidance regarding rainfall would be most welcomed.

2) That a reference point or period was needed as the climate appears to be changing and SSW frequency and intensity were likely to be affected.

3) That the impact of SSW on the Caribbean (especially the Greater Antilles) could be linked to the severity of weather conditions over the eastern regions of mainland USA.

4) That changes in the onset time for seasonal Stratospheric Final Cooling (SFC) and Stratospheric Final

Warming (SFW) may affect rainfall during the Nov-Dec and Apr-May rainfall respectively.

5) Since SSWs are linked to the atmospheric effective temperature and have a positive correlation with cosmic-ray Muon flux (CMF), any short-term increase in cosmic-ray Muon flux is an indication of SSW and any short-term decrease an indication of Sudden Stratospheric Cooling (SSC). Furthermore the SSW, effective temperature and other phenomena which is reflected in the cosmic-ray Muon flux north of $60^{\circ} \mathrm{N}$ are likely to be reflected in the cosmic-ray Muon flux south of $20 \mathrm{~N}$.

To investigate the link between the observed cosmic-ray Muonflux, SSW events and primary dry season rainfall (PDS), an assessment was done over the 
period 1958-2015. Three (3) critical periods were assessed as follows,

1) To create a comprehensive picture of SSW events, a dataset was constructed using the central dates mainly taken from the Japanese 55-year Reanalysis dataset (JRA-55), as quoted in [5]. This dataset covers the period 19582015. Where the SSW central dates were missing in the JRA-55 records, they were supplemented with those from [12]. Three (3) other central dates were taken from the National Ocean and Atmospheric Administration (NOAA) Earth System Research Laboratory (ESRL) and the Physical Science Division dataset (NCEP/NCAR 1). Since the 1961-1990 period was recommended by the WMO for climatological comparisons [36], the dataset was divided in two sections. These two 25-year periods before and after Dec 1990 were extracted from the constructed dataset to represent climate normal periods. These two periods were then examined for the SSW and primary rainfall relationship and the North American Model (NAM) was used to arrive at the duration of the SSW events over the period 2003-2013.

2) The period 2003-2013 was used to assess SSW related severe weather activity over the eastern USA and also for Jamaica. Reagan International Airport (RIA) was used to represent the eastern region of the USA while Sangster International Airport (SIA) was used to represent weather over northern sections of Jamaica during the period. The start date for severe weather impacting RIA in Washington DC USA and SIA in Jamaica was assessed to determine average time from the start of SSW (onset), the end of SSW (cessation) and SSW central dates to the start of significant weather at RIA and SIA.

3) Two empirical assessments of the cosmic-ray Muon observations were done over the period Sep 2011-Sep 2013. These assessments were done to determine whether a pattern or signature existed during the period Nov 2011-Apr 2012 for cosmic-ray Muon flux plus (CMF+) and the Nov 2012-Apr 2013 for the actual cosmic-ray Muon flux (CMF). Sudden Stratospheric Warming (SSW) would be used to signal the start of the event while Sudden Stratospheric Cooling (SSC) would signal the end.

After the frequency tables for the various time slices regarding rainfall and SSWs were generated, correlations were done between the anomalous daily rainfall values and both the CMF+ and CMF periods. The following section 4 outlines the results from these exercises.

\section{Results}

\subsection{Background to Results}

Based on the created SSW database (hereinafter referred to as JRA55+) and the rainfall from the Meteorological Service Jamaica, examined over the period 1958-2015. The results from current study were generated to explore:

- SSW frequency vs Primary Dry Season rainfall.

- Cosmic-ray Muon flux as SSW proxy for predicting primary dry season rainfall. 
- Cosmic-ray Muon flux and primary dry season rainfall correlations.

- Cosmic-ray Muon flux over the Caribbean reflecting apparent SSW event. The following Sections $4.2-4.5$ outline the results of the study.

\subsection{SSW Frequency vs Primary Dry Season Rainfall}

The frequency of the SSW events were generated based on the JRA55+ SSW profile developed. A subset of the JRA55+ record (i.e. 1966-2015) was also used with Dec1990 being the midpoint. The two 25 -year periods which resulted were then assessed and the results outlined in Table 1 below.

Table 1 indicates that the period 1958-2015 had 47 SSW events which occurred in 36 out of the 58-year record. The number of SSW events over the period 1966-90 was also greater than those during the period 1991-2015 however both periods had 16 of the 25 calendar years experiencing SSW events. In those 16 years, 22 SSW events occurred over the 1966-90 period and 19 during the 1991-2015 period. There were no SSWs events recorded between Apr 1992 and Nov 1998 (a six year break) and this could be an area for future investigation as it is the longest in this record. Also noteworthy is the fact that after this period, there was an increase in SSW frequency to almost one every year. The assessment of the rainfall over the island for the period 1958-2015 resulted in 116 seasons examined, when the primary dry season (Nov-April) and wet season (May-Oct) were included. However, over the period Nov 1965-Oct 2015 one hundred (100) seasons were counted of which 50 primary dry seasons were evaluated. After the assessment of the SSW events during the PDS, a pattern emerged that established what was normal. Figure 2 below shows the normal SSW event frequency vs the normal PDS rainfall over the entire period Nov 1957-Apr 2015.

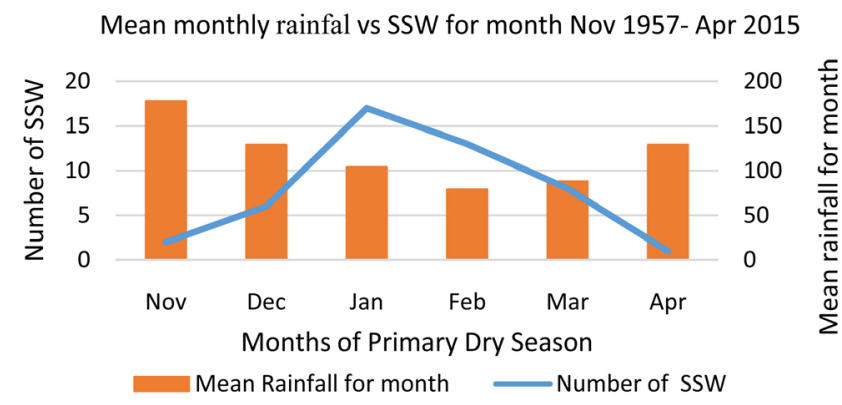

Figure 2. Normal pattern of PDS vs SSW frequency.

Table 1. SSW frequency based on JRA55+.

\begin{tabular}{cccc}
\hline \multicolumn{4}{c}{ Climatological Frequency of Sudden Stratospheric Warming (SSW) } \\
\hline Period & $1958-2015$ & Period 1 $1966-1990$ & Period 2 1991-2015 \\
\hline Number of years & 58 & 25 & 25 \\
Years with SSW events & 36 & 16 & 16 \\
Number of SSW events & 47 & 22 & 19 \\
\hline
\end{tabular}


The assessment concurred with [26] that the highest frequency for SSW was in January and confirmed that the month of lowest rainfall was February. The average rainfall over the 25-year period 1966-90 was greater than the 1991-2015 period. It was also clear that not all PDS experienced SSW events. When the 20-year period1996-2015 was assessed there were 14 PDSs with a total of 18 SSW events while during the previous 20 year (1976-95) period there were 11 PDS periods with $15 \mathrm{SSW}$ events. The other PDSs had no SSWs. This trend suggests increase SSW frequency while the rainfall climatology indicates a reduction in rainfall. This episodic nature of SSW also showed that some calendar years had more than one SSW events with 1981 and 2010 having three (3) SSW events. Nov, Dec and Apr were months with the lowest SSW frequency with SSW rarely occurring in Nov during some decadal assessments. Figure 3 indicates decadal frequency of SSW versus entire PDS rainfall (Nov-Apr).

A review of the literature surrounding Caribbean dry season also found that [32] and [37] included May and November as part of the wet season. Since Nov was also observed as a transition month with rainfall most times significantly above the dry season mean. The focus was shifted to Jan-Mar which was the driest period during the PDS. The assessment of SSW vs Jan-Mar rainfall is outlined in Figure 4 below.

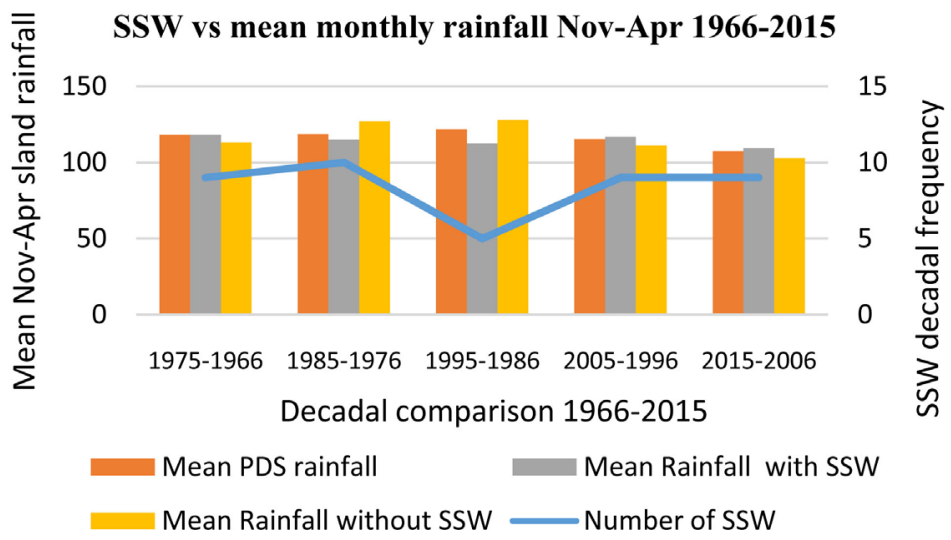

Figure 3. Decadal comparison of SSW versus PDS rainfall.

SSW vs monthly rainfall Jan- Mar periods 1966-2015

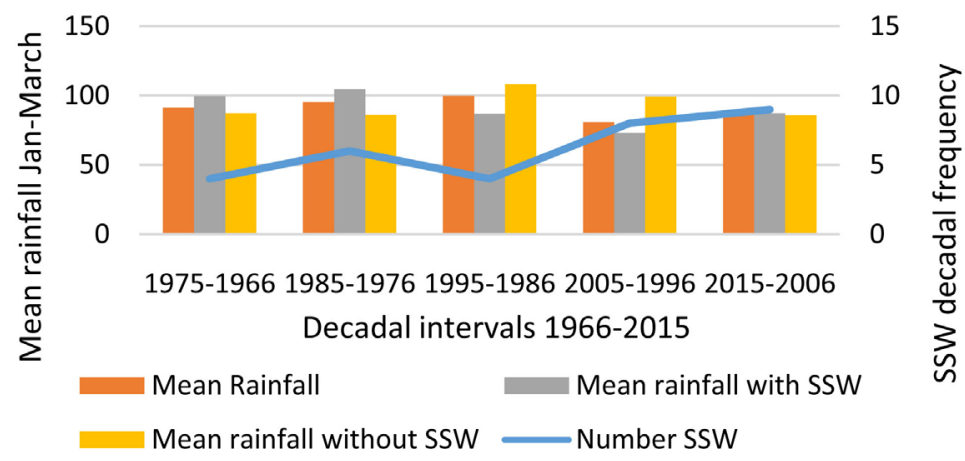

Figure 4. Assessment of SSW and mean island rainfall Jan-Mar 1966-2015. 
When the mean rainfall over the period Jan-Mar was compared with that of Oct-Dec. The Oct-Dec mean rainfall was approximately twice that of the JanMar mean and at times even more. The evaluation over this period showed January was the month with highest SSW frequency while February was the driest month. This again suggested that the months following SSW events are drier. When both periods were assessed the following was observed,

1) Alternate increase then decrease in SSW frequency in successive decades with a pattern change during 2006-2015.

2) A trend towards an increase in the amount of SSW per decade starting 1996-2005.

3) During the period 1966-1985 the Jan-Mar period with SSWs had mean rainfall that was greater than the Jan-Mar periods without SSWs. However, this was reverse from 1986-2005.

During the period 2006-2015 we assessed periods with and without SSW. There was very little difference between the mean rainfall for the periods with SSW and the periods without SSW. Figure 5 below shows a monthly assessment of SSW and rainfall for the months Jan, Feb and Mar over the 1966-2015. When these months were assessed individually a new trend was observed where March was revealed as the driest month.

During the assessment, it was also observed that both Jan and Feb had similar SSW frequency over the period 1966-2015. The trend was shifting to Feb becoming the month with highest SSW frequency during this period. March was the month with 2 or more SSW events occurring in a single month, and was also the month having the lowest amount of SSW events. In this case the low SSW frequency count in March seem to be due to March being at the end of the SSW season and so would naturally have the lowest SSW frequency. March however was not usually drier than February but had emerge as such over the 50-year period 1966-2015. The impact of apparent short duration SSW events during March is also one that merits further study.

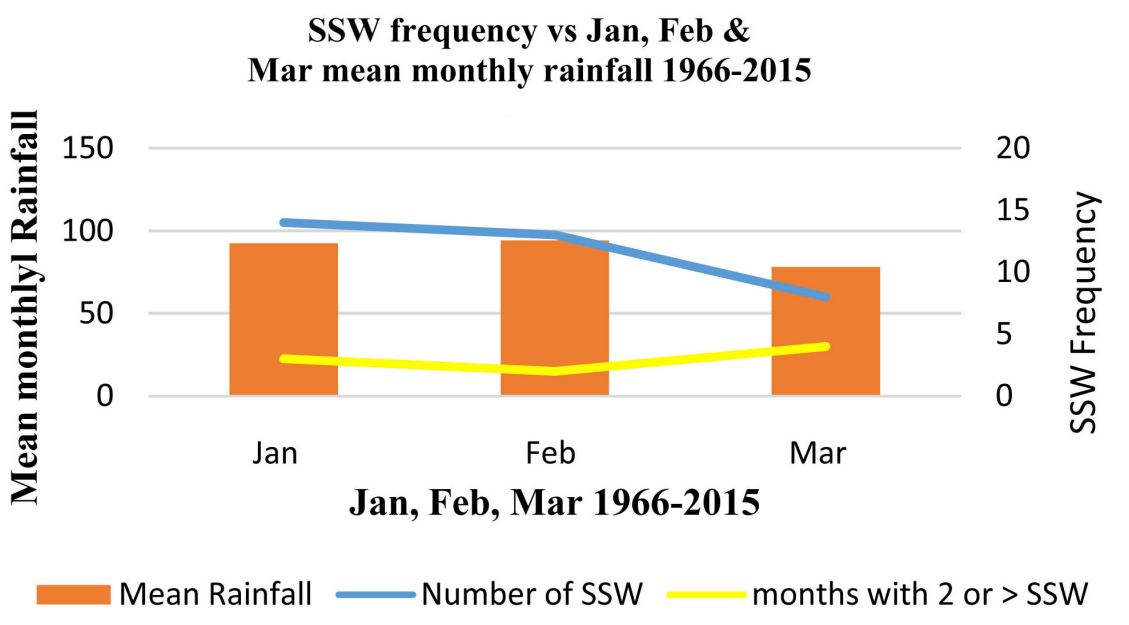

Figure 5. SSW events versus Jan-Mar periods 1966-2015. 


\subsection{Cosmic-Ray Muon Flux as SSW Proxy for Predicting Primary Dry Season Rainfall}

The assumption that an increase in the Cosmic-ray Muon flux (CMF) indicates stratospheric warming due to its positive correlation with the atmospheric effective temperature and that a decrease indicate cooling is central to our approach. The notion therefore is that once the level of cosmic-ray Muon flux increases (decreases) significantly for a sustained period (say more than 2 - 3 days) outside of proven ground level enhancement (GLE) or For bush Decrease (Fd), this would signal the start (end) of the SSW. The increases were deemed as SSW, while the decreases marked SSC. This is based on work done at mid-high latitudes by [19] and [20] at the South Pole. [38] using the Weakly Ionizing LeadLepton Interaction (WILLI) detector gives a clear indication of the likely levels of variation for GLE, Fds and other non-meteorological factors that can cause variation of cosmic-ray Muon flux. Using ten (10) SSW events over the period 2003-2013, we assessed the time lapse between the start of SSW events, the end of SSW events, the SSW central dates and to the start of severe weather over Reagan International Airport (RIA) and Sangster international Airport (SIA). Of these $10 \mathrm{SSW}$ events it was observed that most of the related significant weather events occurred after SSW started both over RIA and SIA. Significant weather after the start of SSW affects Jamaica on average 18 days after affecting RIA. We also observed that after the end of these SSW events significant rainfall occurred over Jamaica on average 7 days after RIA. When the central dates were used significant changes in rainfall at SIA occurred on average 13 days after RIA. Other results from this assessment are shown in Table 2 below.

Table 2 contains the average number of days and the number of events used in its calculation. The assessment revealed that although there were occasions when severe weather occurred over RIA for the entire duration of SSW events, it was observed that on average, significant change in weather occurred 15 days after the central dates. Significant weather events which occur before the start of SSW were ruled out as being unassociated events. The average amount of days from SSW start to significant weather over Jamaica was 39 days while from the end of SSWs it was 15 days. Also notable was the time from the central date which was on average 29 days to the start of significant weather over SIA. The guidance therefore that cosmic-ray Muon flux can be used as a proxy to identify

Table 2. Average time lapse from SSW to significant weather over SIA and RIA.

\begin{tabular}{ccccccc}
\hline Location & \multicolumn{2}{c}{$\begin{array}{c}\text { Significant weather } \\
\text { from start of SSW event }\end{array}$} & \multicolumn{2}{c}{$\begin{array}{c}\text { Significant weather } \\
\text { from end of SSW event }\end{array}$} & \multicolumn{2}{c}{$\begin{array}{c}\text { Significant weather } \\
\text { start from Central Date }\end{array}$} \\
\hline Airports & $\begin{array}{c}\text { Ave days from } \\
\text { start of SSW }\end{array}$ & $\begin{array}{c}\text { Number } \\
\text { of SSW } \\
\text { events }\end{array}$ & $\begin{array}{c}\text { Ave days } \\
\text { from End } \\
\text { of SSW }\end{array}$ & $\begin{array}{c}\text { Number } \\
\text { of SSW } \\
\text { events }\end{array}$ & $\begin{array}{c}\text { Ave days from } \\
\text { Central Date }\end{array}$ & $\begin{array}{c}\text { Number } \\
\text { of SSW } \\
\text { events }\end{array}$ \\
\hline RIA & 21 & 6 & 8 & 2 & 15 & 6 \\
SIA & 39 & 7 & 15 & 6 & 29 & 7 \\
\hline
\end{tabular}


SSW. This therefore would suggest that on average 39 days after a sustained increase in cosmic-ray muon flux (SSW) or 15 days after a sustained decrease in cosmic-ray Muon flux (SSC) Jamaica is likely to experience a significant change in weather during the primary dry season.

\subsection{Cosmic-Ray Muon Flux and Primary Dry Season Rainfall Correlations}

The correlation between PDS rainfall and cosmic-ray Muon flux over Jamaica was examined using three rainfall indices. The first index was the actual daily rainfall for Sangster International Airport (SIA), the second was for the daily rainfall for Norman Manley International Airports (NMIA) and the third a simple average of both SIA and NMIA rainfall. Data from these two stations represented the most consistent dataset gathered by WMO certified observers at major airports in Jamaica. The results for the period Nov 2011-Apr 2012 and Nov 2012-Apr 2013 were generated. The correlation values for the Nov 2012 to Apr 2013 (CMF) are shown in Table 3 below. The results for the control period $\mathrm{CMF}+$ were similar but of opposite sign.

Table 3 represents lag correlation values between the rainfall at SIA and SIA/NMIA-average over a 48 hour period. SIA, being on the north side of the island, was more severely impacted by weather systems such as cold fronts and troughs moving from over the eastern sections of the USA. A significant decrease in correlation values was observed after 3-day lag while the 0 - 48 hour lag had no significant change in correlation values. The cosmic-ray Muon flux was negatively correlated with PDS rainfall with the strongest values being -0.40 (0600Z) and the lowest -0.03 (1200Z).

\subsection{Cosmic-Ray Muons UWI Mona during Reported SSW North of $60^{\circ} \mathrm{N}$}

In this section the results are based on an empirical examination of the SSW events during Jan 3-Feb 2, 2012 and Jan 5-Feb 2, 2012 based on work done by [19] and [20] who showed that cosmic-ray Muon flux reflect the atmospheric effective temperature, and that the cosmic-ray Muon flux pattern associated with reported SSW north of $60^{\circ} \mathrm{N}$ was also reflected in cosmic-ray Muon flux measured at the UWI Mona. This we theorize may not always be the case but is most

Table 3. Cosmic-ray Muon flux vs daily rainfall from selected stations over Jamaica.

\begin{tabular}{ccccc}
\hline Lag periods & \multicolumn{2}{c}{24 hour lag } & \multicolumn{2}{c}{48 hour lag } \\
\hline Station & SIA/NMIA & SIA & SIA/NMIA & SIA \\
\hline MuFlux06Z & -0.38 & -0.40 & -0.32 & -0.33 \\
MuFlux12Z & -0.11 & -0.12 & -0.04 & -0.03 \\
MuFlux18Z & -0.32 & -0.34 & -0.39 & -0.39 \\
MuFlux00Z & -0.30 & -0.34 & -0.38 & -0.38 \\
\hline
\end{tabular}


likely so during major SSW events. The duration of SSWs was justified based on not only reviews of the literature, but from the stratospheric temperature reports and the use of the North American numerical weather prediction model (NAM). Figure 6 below shows UWI Mona cosmic-ray Muon flux plus (CMF+) anomalies when the mean CMF+ over the primary dry season period was subtracted from the actual observations. The period shown encapsulates the SSW event of Jan 3-Feb 2, 2012.

The CMF+ data record suggests a warming period that started Dec 24, 2011 and ended Jan 3, 2012 which indicate an 8-day warming before the reported SSW event started. The analysis of the anomalies also suggests that cooling started on Jan 3 and ended Jan 8, 2012. This therefore represented a warming over a ten (10) day period and a cooling period over 5 days, which overlapped into the SSW event. A similar warming and cooling period was observed during the following PDS (winter) and this is indicated in Figure 7 below, which encapsulates the SSW event.

The trend in cosmic-ray Muon flux over the entire study showed a general decrease. However, the increase shown in both Figure 6 and Figure 7 was

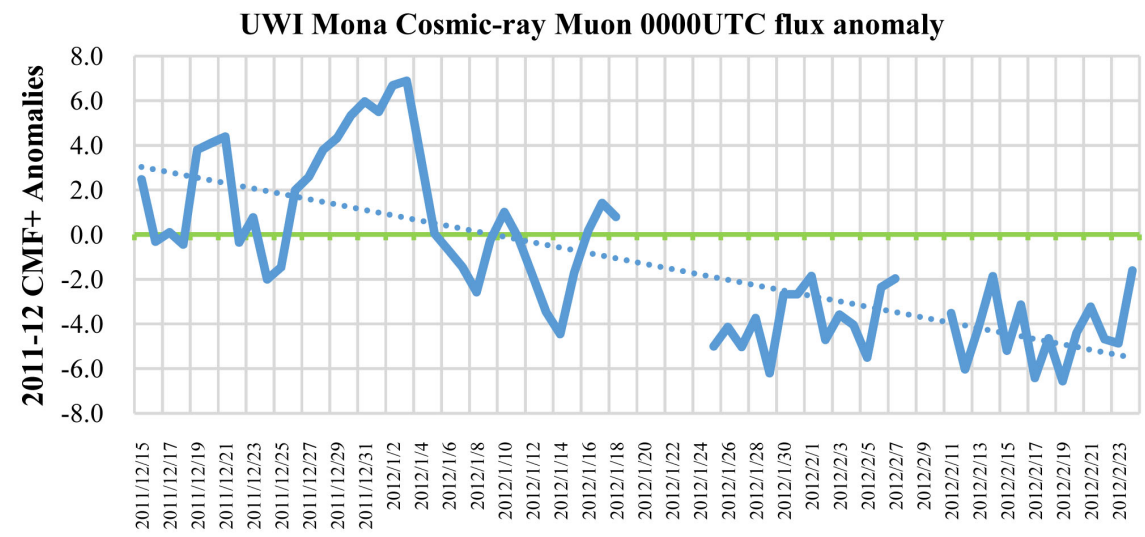

SSW event Capture period 2011-12

Figure 6. CMF+ anomalous value plot reflecting SSW event Jan 3 to Feb 2, 2012.

UWI Mona Coamic-ray Muon 0000 UTC flux anomaly

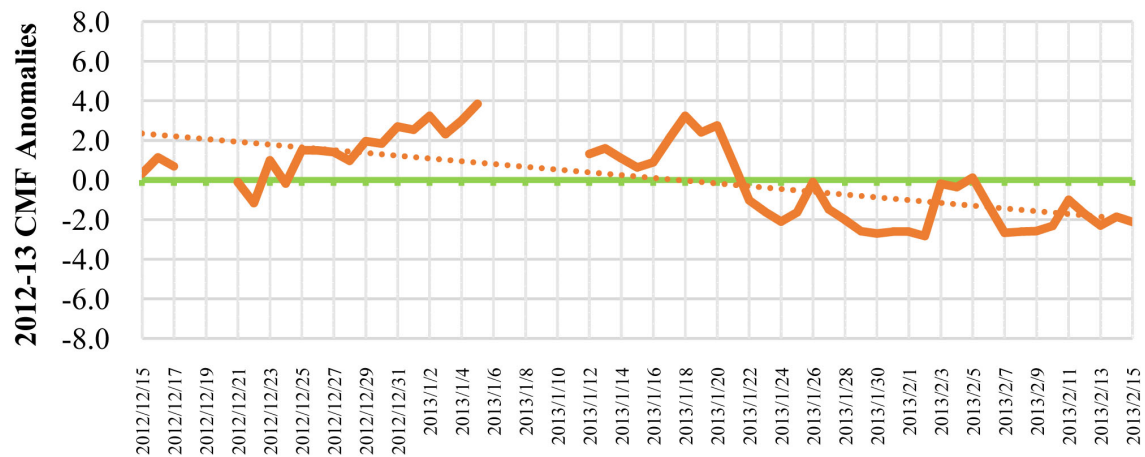

SSW event Capture period 2012-13

Figure 7. CMF anomalous value plot reflecting SSW event Jan 5 to Feb 3, 2013. 
significantly above the current trend. The cosmic-ray Muon flux (CMF) over several days, starting about Dec 23, 2012 associated positive anomalies, remained until January 21, 2013. This represented a 29 day warming period which was followed by a two (2) day period of cooling before the flux returned to its downward trend. While further research is recommended, the empirical results suggest that both the cosmic-ray Muon flux plus and the actual cosmic-ray Muon flux recorded are sensitive to changes during the primary dry season especially when SSW events occurred. The CMF+ plus showed changes before the SSW event while the CMF showed SSW-type changes before and during the event.

\section{Summary}

Cosmic ray Muons are sub-atomicparticles formed when cosmic radiation interact with molecules within the Earth's atmosphere. This interaction initially takes place in the lower stratosphere resulting in formation of other Pions and Kaons, which decay almost immediately to Muons. Sudden stratospheric warming is a sharp increase in the stratospheric temperature which has been found to have a positive correlation to cosmic-ray Muon flux. When major SSW events occur, they result in a change in the wind direction and speed associated with the Polar Vortex. Consequently, Arctic air moves southwards over North America resulting in storms and blizzards that sometimes affect eastern regions of the USA. When this happens the frequency and intensity of severe weather events from high pressure systems, frontal systems and troughs over the northwestern Caribbean which include Jamaica increases. The period with highest frequency for major SSWs was Jan-Mar with Jan being the month having the highest amounts. The driest period of the Jamaican primary dry season is also Jan-Mar with February as the driest. When the months Jan, Feb and Mar were assessed individually between 1966 and 2015 it revealed that the number of SSWs in Jan was almost the same as in Feb and that the month with the lowest rainfall amounts was March.

Based on the documented positive correlation which exist between cosmic-ray Muon flux at latitudes north of $60^{\circ} \mathrm{N}$ and sudden changes in stratospheric temperature, the assumption was made that this is likely to be reflected in the cosmic ray Muon flux at low latitude. Therefore a comparison between SSW events and similar periods of the cosmic-ray Muon flux recorded at the UWI Mona Campus was done. When the anomalous cosmic-ray Muon flux that was matched with SSW event period Jan 3-Feb 2, 2012 a 10-day warming period was observed before the report SSW event and a 5-day cooling period after. However, this comparison also revealed an increase in cosmic ray Muon flux at UWI Mona during the SSW event of Jan 5-Feb 32013 by 18 days.

During the assessment of correlation between PDS rainfall and CMF, an inverse correlation was generated between station averaged rainfall (SIA + NMIA - average) and also SIA by itself. The highest correlation value range when the SIA rainfall was -0.34 to -0.40 for the zero lag and -0.33 to -0.39 for the 24 
hour lag. This indicates that there was not much change in correlation values over time, with most being moderate but robust.

When the various SSW associated dates were evaluated for links to significant changes in weather, it was found that on average 29 days after the central date a significant change in rainfall was likely over SIA Jamaica. Significant rainfall was also likely over SIA on average 15 days after the end of SSW event. The RIA which represents the eastern USA, was likely to experience significant changes in weather on average 21 days from the start of the SSW and 15 days from central dates. In Section 6 below, we outline the conclusions drawn from this study.

\section{Conclusions}

[3] argued that very little is known regarding the drivers of rainfall anomalies for the primary dry season and suggested that this is an area that merits much more research. [3] also argued that significant differences exist between the inter-annual variability of the early versus the late Caribbean rainfall season and that using climate phenomena such as sea surface temperature anomalies (SSTA) only explains part of this variability. In this study, we investigate the link between cosmic ray Muon flux and SSW (a winter warming phenomenon which is typically observed north of $60^{\circ} \mathrm{N}$ ), to determine whether it extends to the Caribbean. Our aim was to was to determine if it can be identified routinely in cosmic-ray Muon flux this far south and used for forecasting purposes. This is based on work done by [19] and [20] who have shown that there is a positive correlation between cosmic-ray Muon flux and SSWs. We therefore suggest that from this initial work, this El Nino-type stratospheric phenomenon can be monitored by cosmic-ray muon flux from low-cost Muon detectors routinely over the Caribbean and consequently submit the following findings.

1) The JRA55 + SSW profile created for this study confirmed January to be the month of greatest SSW frequency and that the month of February was the driest month during Jamaica's primary dry season. This suggests a pattern where the driest month follows the month of highest SSW frequency and the projected increase in SSW for February points to a reduction in March rainfall. We therefore suggest that the cosmic-ray Muon flux which has a positive correlation to SSW via the atmospheric effective temperature, can also be used to monitor rainfall over the long term.

2) The results suggest that after the end of SSW events, a significant change in weather is likely over Jamaica on average after 29 days and alsoon average 15 days after central date. We therefore suggest were practical guidancefor using cosmic-ray Muon flux to track the occurrence of SSWs to make rainfall projections.

3) The highest correlation values for cosmic-ray Muon flux (CMF) when lagged against rainfall at the Sangster International Airport (SIA) for the first day (0 lag) are -0.4 using 0600 UTC cosmic-ray Muon flux and -0.39 using 1800 UTC (24 hr lag). This suggests modest but robust values that will benefit from further research as data increases over the region. 
4) In both cases where the cosmic-ray Muon Flux plus (the unfiltered data with $50 \%-70 \%$ Muons) and the actual cosmic ray Muon flux was used, there was significant alignment with SSW event reported north of $60^{\circ} \mathrm{N}$. Although the periods compared did not behave identically we suggest that the cosmic-ray Muon flux is sensitive to changes in stratosphere temperature fluctuations at low latitudes and merit further research.

Knowledge of significant variations in the island's rainfall during the PDS has become even more important for major players in the Jamaican economy regarding such areas as tourism, agriculture and other end user applications, [39]. This is especially so for tourism which is our primary foreign exchange earner and is directly correlated to the severity of the North American winter. Predicting anomalous rainfall during the primary dry season linked to SSW events by monitoring cosmic-ray Muon flux over the Caribbean therefore has potential and merits significant further attention.

\section{Acknowledgements}

Stephen Silvera and Andre Gordon for their assistance with data capture and advise.

\section{Conflicts of Interest}

The authors declare no conflicts of interest regarding the publication of this paper.

\section{References}

[1] Hastenrath, S.L. 1967) A Contribution to the Wind Conditions over the Caribbean Sea and Gulf of Mexico. Tellus, 20, 163-178. https://doi.org/10.3402/tellusa.v20i1.9996

[2] Magana, V., Amador, J.A. and Medina, S. 1999) The Midsummer Drought over Mexico and Central America. American Meteorological Society, Boston, MA. https://doi.org/10.1175/1520-0442(1999)012<1577:TMDOMA >2.0.CO;2

[3] Stephenson, T.S., chen, A.A. and Taylor, M.A. (2007) Toward the Development of Prediction Models for the Primary Caribbean Dry Season. Theoretical and Applied Climatology, 92, 87-101. https://doi.org/10.1007/s00704-007-0308-2

[4] Limpasuvan, V., Thompson, D.W.J. and Hartmann, D.L. (2004) The Life Cycle of the Northern Hemisphere Sudden Stratospheric Warmings. Journal of Climate, 17, 2584-2597. https://doi.org/10.1175/1520-0442(2004)017<2584:TLCOTN>2.0.CO;2

[5] Baldwin, M.P. and Dunkerton, T.J. (1999) Propagation of the Arctic Oscillation from the Stratosphere to the Troposphere. Journal of Geophysical Research: Atmospheres, 104, 30937-30946. https://doi.org/10.1029/1999JD900445

[6] Baldwin, M.P. and Dunkerton, T.J. (2001) Stratospheric Harbingers of Anomalous Weather Regimes. Nature, 294, 581-584. https://doi.org/10.1038/news011025-4

[7] Butler, A.H., Seidel, D.J., Hardiman, S.C., Butchart, N., Birner, T. and Match, A. (2015) Defining Sudden Stratospheric Warmings. Bulletin of the American Meteorological Society, 96, 1913-1928. https://doi.org/10.1175/BAMS-D-13-00173.1

[8] Nath, D., Chen, W., Zelin, C., Pogoreltsev, A.I. and Wei, K. (2016) Dynamics of 
2013 Sudden Stratospheric Warming Event and Its Impact on Cold Weather over Eurasia: Role of Planetary Wave Reflection. Scientific Reports, 6, 24174. https://doi.org/10.1038/srep24174

[9] Hocke, K., Lainer, M. and Schanz, A. (2015) Composite Analysis of a Major Sudden Stratospheric Warming. Angea Communicates, 33, 783-788. https://doi.org/10.5194/angeo-33-783-2015

[10] Waugh, D.W., Sobel, A.H. and Polvani, L.M. (2017) What Is the Polar Vortex and How Does It Influence Weather? Bulletin of the American Meteorological Society, 98, 37-44. https://doi.org/10.1175/BAMS-D-15-00212.1

[11] Charlton, A.J. and Polvani, L.M. (2007) A New Look at Stratospheric Sudden Warmings. Part I: Climatology and Modeling Benchmarks. American Meteorological Society, Boston, MA. https://doi.org/10.1175/JCLI3996.1

[12] Mitchell, D.M., Gray, L.J., Anstey, J., Baldwin, M.P. and Charlton-Perez, A.J. (2013) The Influence of Stratospheric Vortex Displacements and Splits on Surface Climate. Journal of Climate, 26, 2668-2682. https://doi.org/10.1175/JCLI-D-12-00030.1

[13] Chen, A.A. and Taylor, M.A. (2002) Investigating the Link between Early Season Caribbean Rainfall and the El Niño + 1 Year. International Journal of Climatology, 22, 87-106. https://doi.org/10.1002/joc.711

[14] Jury, M., Malmgren, B.A. and Winter, A. (2007) Subregional Precipitation Climate of the Caribbean and Relationships with ENSO and NAO. Journal of Geophysical Research, 112. https://doi.org/10.1029/2006JD007541

[15] Charlery, J., Nurse, L. and Whitehall, K. (2006) Exploring the Relationship between the North Atlantic Oscillation and Rainfall Patterns in Barbados. International Journal of Climatology, 26, 819-826. https://doi.org/10.1002/joc.1334

[16] Taylor, M.A., Enfield, D.B. and Chen, A.A. (2002) Influence of the Tropical Atlantic versus the Tropical Pacific on Caribbean Rainfall. Journal of Geophysical Research, 107, 3127. https://doi.org/10.1029/2001JC001097

[17] Hu, J.G., Ren, R.C. and Xu, H.M. (2014) Occurrence of Winter Stratospheric Sudden Warming Events and the Seasonal Timing of Spring Stratospheric Final Warming. Journal of the Atmospheric Sciences, 71, 2319-2334. https://doi.org/10.1175/JAS-D-13-0349.1

[18] Chernov, D.V, Barbashina, N.S., Mannocchi, G., Kokoulin, R.P., Kompaniets, K.G., Petrukhin, A.A., Saavedra, O., Shutenko, V.V., Timashkov, D.A. and Trinchero, G. (2005) Experimental Setup for Muon Diagnostics of the Earth's Atmosphere and Magnetosphere (the URAGAN Project). 29 th International Cosmic Ray Conference Pune, Pune, India, 457-460.

[19] Osprey, S., Barnett, J., Smith, J., Adamson, P., Andreopoulos, C., Arms, K.E., Armstrong, R., Auty, D.J., Ayres, D.S. and Baller, B. (2009) Sudden Stratospheric Warmings Seen in MINOS Deep Underground Muon Data. Geophysical Research Letters, 36, 1-18. https://doi.org/10.1029/2008GL036359

[20] Tilav, S., Desiati, P., Kuwabara, T., Rocco, D., Rothmaier, F., Simmons, M. and Wissing, H. (2010) Atmospheric Variations as Observed by IceCube.

[21] Maghrabi, A., Almutayri, M., Al-Harbi, H. and Baig, M. (2015) Atmospheric-Weighted Temperature and Its Influence on Cosmic Ray Muons. The 34th International Cosmic Ray Conference, Hague, Netherlands, 30 July-6 August 2015, 1-8. https://doi.org/10.22323/1.236.0040

[22] Zhang, X. (2016) Statistical Modeling of Effective Temperature with Cosmic Ray Flux. Georgia State University, Atlanta, GA.

https://scholarworks.gsu.edu/phy_astr_diss/85 
[23] Kniveton, D.R. and Todd, M.C. (2001) On the Relationship of Cosmic Ray Flux and Precipitation. Geophysical Research Letters, 28, 1527-1530. https://doi.org/10.1029/2000GL012536

[24] Pedatella, N.M., Chau, J.L., Schmidt, H., Goncharenko, L.P., Stolle, C., Hocke, K., Harvey, V.L., Funke, B. and Siddiqui, T.A. (2018) How Sudden Stratospheric Warming Affects the Whole Atmosphere. https://doi.org/10.1029/2018EO092441

[25] Tripathi, O.P., Baldwin, M., Charlton-Perez, A., Charron, M., Eckermann, S.D., Gerber, E., Harrison, R.G., Jackson, D.R., Kim, B. and Kuroda, Y. (2015) The Predictability of the Extratropical Stratosphere on Monthly Time-Scales and Its Impact on the Skill of Tropospheric Forecasts. Royal Meteorological Society, 141, 987-1003. https://doi.org/10.1002/qj.2432

[26] Butler, A.H., Sjoberg, J.P., Seidel, D.J. and Rosenlof, K.H. (2016) A Sudden Stratospheric Warming Compendium. Earth System Science Data, 9, 63-76. https://doi.org/10.5194/essd-2016-49

[27] Scaife, A.A., Yu Karpechko, A., Baldwin, M.P., Brookshaw, A., Butler, A.H., Eade, R., Gordon, M., MacLachlan, C., Martin, N., Dunstone, N. and Smith, D. (2016) Seasonal Winter Forecasts and the Stratosphere. Atmospheric Science Letters, 17, 51-56.

[28] Nigam, S. and Baxter, S. (2015) Teleconnections. In: Encyclopedia of Atmospheric Sciences, Elsevier, Amsterdam, 90-109. https://doi.org/10.1016/B978-0-12-382225-3.00400-X

[29] Mok, H.M. and Cheng, K.M. (2001) The Day-Night Variation of Cosmic Ray Intensity at Sea Level under the Influence of Fronts and Troughs.

[30] Poirier, J. and Catanach, T. (2011) Atmospheric Effects on Muon Flux at Project GRAND. 2011 32nd International Cosmic Ray Conference, Beijing, 11-18 August 2011.

[31] Marusek, J.A. (2006) The Relationship between Cosmic Rays and Hurricanes James A. Marusek. 2nd International Conference on Global Warming and the Next Ice Age, 1-6.

[32] Barbashina, N.S., Borog, V.V., Dmitrieva, A.N., Kokoulin, R.P., Kompaniets, K.G., Petrukhin, A.A., Timashkov, D.A., Shutenko, V.V. and Yashin, I.I. (2007) Muon Diagnostics of the Earth's Atmosphere and Magnetosphere. Bulletin of the Russian Academy of Sciences. Physics, 71, 1041-1043. https://doi.org/10.3103/S1062873807070441

[33] Yashin, I.I., Astapov, I.I., Barbashina, N.S., Borog, V.V., Dmitrieva, A.N., Chernov, D.V., Kokoulin, R.P., Kompaniets, K.G., Mishutina, Y.N. and Petrukhin, A.A. (2015) Real-Time Experimental Data of the Muonhodoscope URAGAN Accessible in www. Journal of Physics: Conference Series, 632, Article No. 012086. https://doi.org/10.1088/1742-6596/632/1/012086

[34] Rylander, J., Jordan, T. and Paschke, J. (2010) QuarkNet Cosmic Ray Muon Detector User's Manual.

[35] Lofgren, J. (2001) QuarkNet Cosmic Ray Detector System. Cooperative Engineering Education IV, Valparaiso University, Valparaiso, IN.

[36] WMO-No 1203 (2017) WMO Guidelines on the Calculation of Climate Normals. In Purpose. Chairperson, Publications Board World Meteorological Organization (WMO), Geneva, Switzerland.

[37] Hastenrath, S.L. (1990) The Relationship of Highly Reflective Clouds to Tropical Climate Anomalies. Journal of Climate, 3, 353-365.

https://doi.org/10.1175/1520-0442(1990)003<0353:TROHRC >2.0.CO;2 
[38] Saftoiui, A., Bercuci, A., Brancus, I.M., Duma, M., Haungs, A., Mitrica, B., Petcu, M., Rebel, H., Sima, O. and Toma, G. (2010) Measurement of the Cosmic Muon Flux with the WILLI Detector as a Source of Information about Solar Events.

[39] WMO-No. 100 (2011) Guide to Climatological Practices. In: Uses of Climatological Information and Research, World Meteorological Organization, Geneva, Switzerland. 